\title{
Es wird normaler, anders zu sein
}

\section{Daniel Lüthi}

Freier Journalist, Medientrainer Bern

«Menschen mit Behinderung in der Welt 2035»: Dies der Titel einer Studie, die das Gottlieb Duttweiler Institut (GDI) im Auftrag der Stiftung Cerebral durchgeführt und in Rüschlikon vorgestellt hat.* Fazit: Viele Barrieren sind schon verschwunden, weitere werden folgen. Neue Technologien und medizinische Fortschritte helfen dabei. Besonders schwierig zu überwinden sind jedoch gesellschaftliche Hürden.

«Die Schweizer leben in der besten aller Welten»: So unverschämt positiv war bei der Präsentation der Studie die Zwischenbilanz von David Bosshard, CEO des GDI. Er meinte damit explizit die behinderten Menschen in der Schweiz, und die Fortschritte, die hierzulande bereits erzielt worden sind auf ihrem Weg zu mehr Autonomie und damit zur Inklusion, also zu einer umfassenden gesellschaftlichen Teilhabe.

Immer mehr seien aus Schicksalen «Machsale` geworden, die Fortschritte in der Medizin punkto Diagnostik und Therapien hätten dazu wesentliche Beiträge geleistet. Gestiegen seien parallel dazu aber auch die Ansprüche und Erwartungen, bei Behinderten und in der übrigen Gesellschaft; gerade in der Arbeitswelt habe der Druck zugenommen. Eher verhalten daher Bosshards Blick nach vorn: «Wir dürfen nicht überoptimistisch sein.»

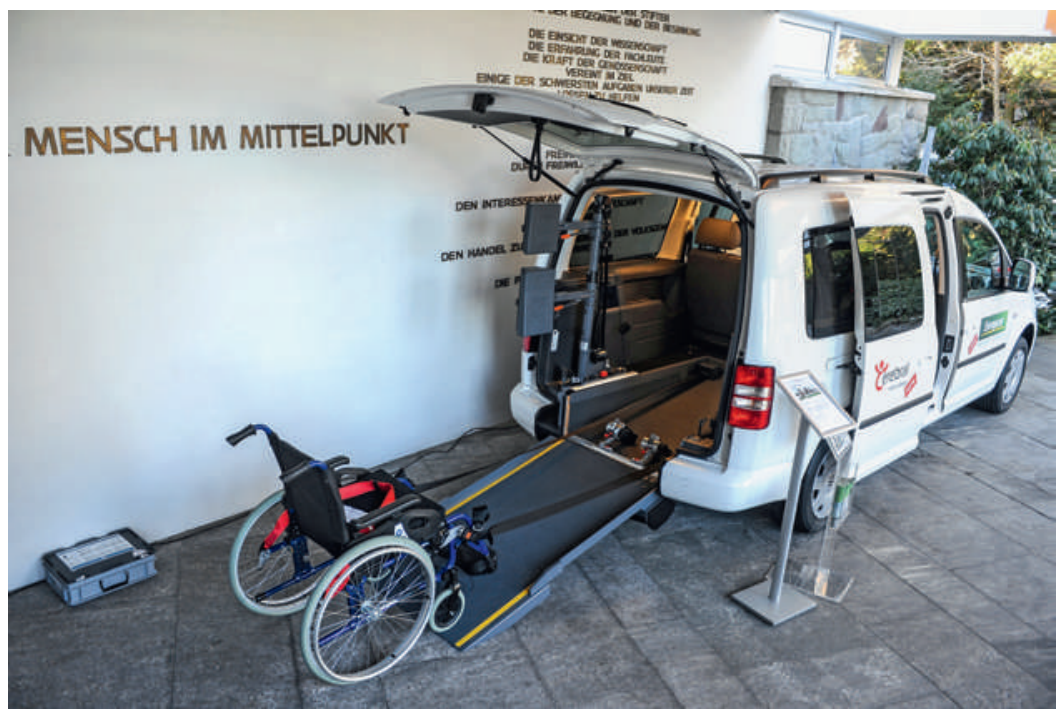

Körperliche Einschränkungen - insbesondere bei der Mobilität - und die entsprechenden Hilfsmittel prägten in Rüschlikon das Bild.

\section{Die Resultate der Studie}

Optimistisch sein dürfen wir, heisst das ja auch. Mirjam Hauser, Co-Autorin der Studie, bestätigte diese Sichtweise. Der Blick nach vorn, der aus ihren Recherchen resultiere, sei "positiv gestimmt", sagte sie in Rüschlikon. "Wir sind auf dem richtigen Weg - aber das dauert.»

Konkret fasst das GDI seine Erkenntnisse in sechs Punkten zusammen:

1. Behindertensein wird normaler. Menschen mit Behinderungen werden präsenter, gesellschaftliche Vorurteile werden weiter abgebaut. Aber: «Statusstress und ein generell egozentrischeres Weltbild bremsen diese Entwicklung. Gefordert sind gezielte Massnahmen, um Stigmatisierungen entgegenzutreten.»

2. Barrieren verschwinden. Bis 2024 muss der öffentliche Raum barrierefrei sein, so will es das Gesetz. «In zehn Jahren bringt also vielleicht der SelfDriving-Car von Google auch Seh- oder Gehbehinderte ans gewünschte Ziel.»

3. Neue Wohnformen entstehen. Gemeinschaftliches Wohnen wird wichtiger, «ein grösseres Angebot an dezentralen Wohnformen führt zu fluideren Wohnwelten. Zwischen Heim und Daheim gibt es vielfältige neue Zwischenstufen - mit Services je nach Bedarf.»

4. Technologie flexibilisiert Pflege. «Neue Pflegetechnologien und Rehabilitationsroboter werden eine flexible ambulante Betreuung und Pflege ermöglichen. Neuartige Technologien übernehmen Basispflegeleistungen und schaffen Raum für Menschlichkeit - trotz Fachkräftemangel.»

5. Arbeitsmarkt wird solidarischer - und härter. Der zunehmende Druck polarisiert: Auf der einen Seite globale Unternehmen, "welche die flinksten Ta- 
lente anziehen, auf der anderen Seite Aussteigerunternehmen, die auf «Slow ist beautiful und lokale Wertschöpfung setzen.» Die Mitte geht verloren.

6. Neue Inklusionsansätze reformieren die Bildungswelt. «Die Grenzen zwischen normal und behindert verschwimmen immer mehr", heisst es in der Studie. Gerade an den Schulen und in der Ausbildung aber gibt es bezüglich Toleranz und Akzeptanz noch besonders viel zu tun, sagten Betroffene dazu.

\section{Körperliche und andere Behinderungen}

Körperliche Einschränkungen - insbesondere bei der Mobilität - und die entsprechenden Hilfsmittel prägten in Rüschlikon das Bild, was sicher in einem direkten Zusammenhang mit der Stiftung Cerebral stand, welche die Studie in Auftrag gegeben hatte. Rollstuhlfahrer waren nicht nur im Publikum recht prominent vertreten, sondern auch auf der Leinwand: Winter- und Spitzensport trotz allem waren Themen, die gerade technische Errungenschaften eindrücklich belegten.

Speziell ist, dass am Tag, bevor das GDI seine Studienergebnisse präsentierte, der Bundesrat bekannt gegeben hatte, nach welchen Leitlinien er die Invaliden-

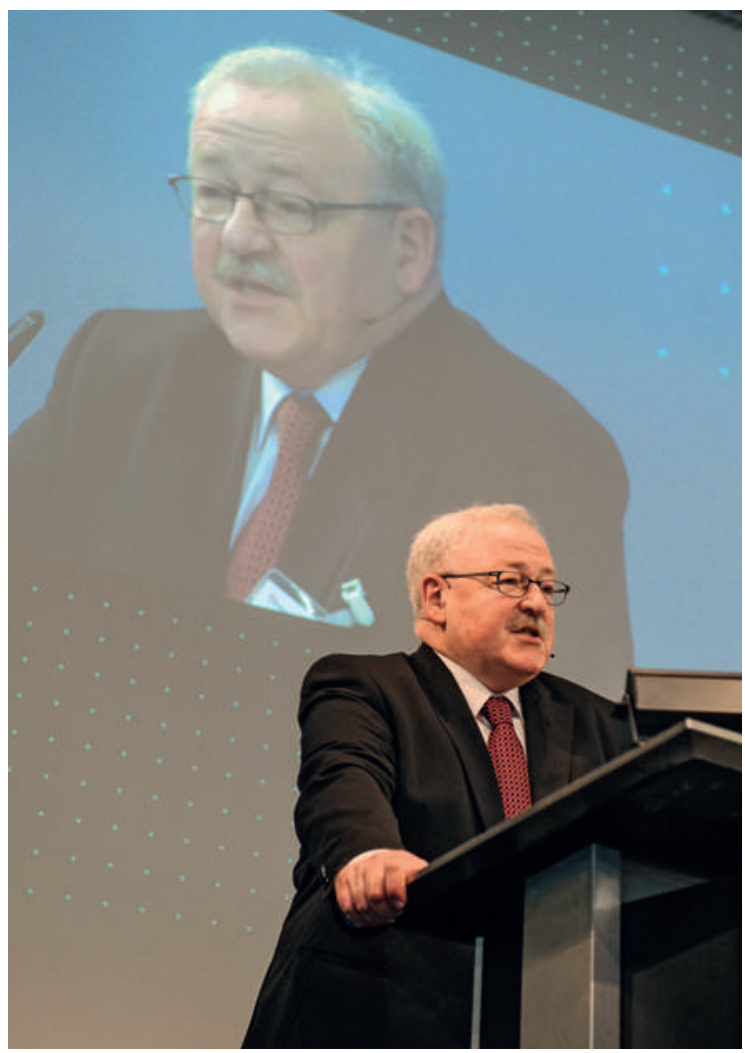

Jürg Brechbühl, Direktor des Bundesamtes für Sozialversicherungen.

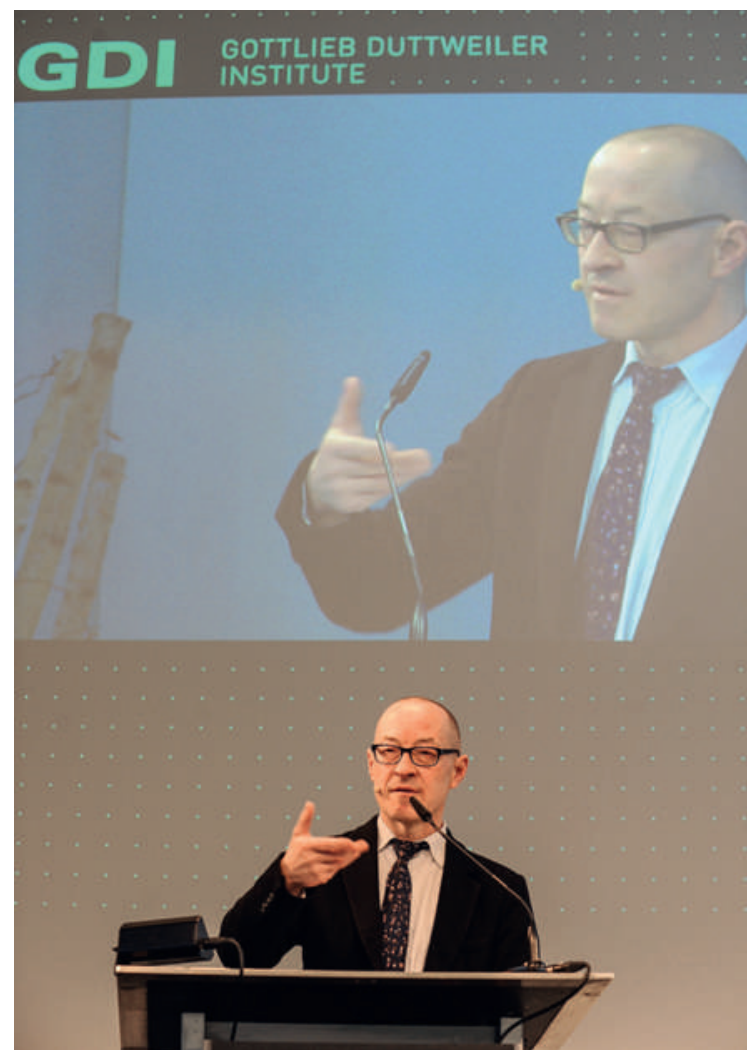

David Bosshard, CEO des Gottlieb Duttweiler Instituts.

versicherung IV reformieren will. Er richtet sein Augenmerk vor allem auf junge Menschen - und auf psychisch Kranke. Jürg Brechbühl, Direktor des Bundesamtes für Sozialversicherungen, bestätigte in Rüschlikon diese Stossrichtung. Gegenüber der SÄZ sagte er: «Vor allem bei der Integration von Personen mit einer körperlichen Behinderung haben wir in den vergangenen Jahren grosse Fortschritte gemacht. Aber bei 18- bis 24-Jährigen und Menschen mit psychischen Problemen - immerhin fast die Hälfte aller IV-Bezüger - haben wir noch viel zu wenig erreicht. Hier wollen wir die Leistungen der IV gezielt verbessern. Wir investieren in Beratung und Coaching nicht nur für Versicherte, sondern auch für Arbeitgeber.»

Brechbühl unterstrich damit einen Eindruck, den die Tagung in Rüschlikon zum Teil unausgesprochen bekräftigte: dass «Behinderung» oft auf körperliche Behinderung und ihre sichtbaren Symbole wie den Rollstuhl reduziert wird, dass andere - darunter eben auch psychische - Einschränkungen in der Wahrnehmung und bezüglich Massnahmen jedoch nach wie vor vernachlässigt werden.

Konrad Widmer, Psychiater und Präsident der universitären psychiatrischen Kliniken Basel, sagte es in Rüschlikon gegenüber der SÄZ so: «Psychische Behinderungen sind oft nicht sichtbar und deshalb 


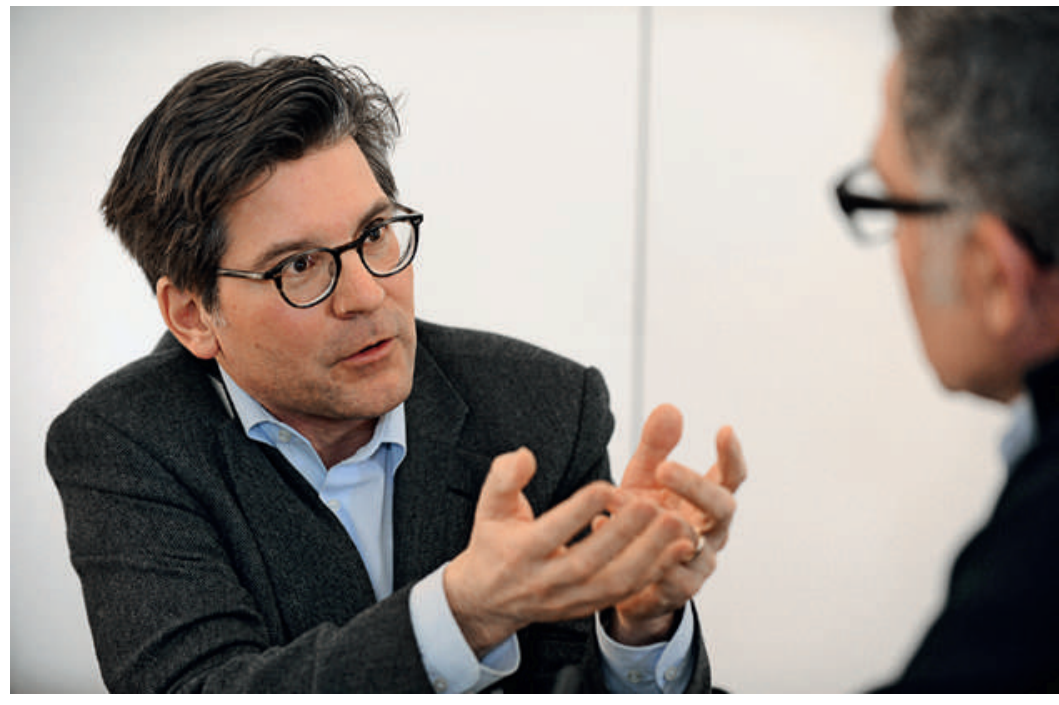

Die SÄZ im Gespräch mit Andreas Meyer-Heim, medizinischer Leiter der Abteilung Rehabilitation am Universitäts-Kinderspital Zürich.

besonders schwer zu verstehen. Sie werden deshalb immer noch speziell stigmatisiert. Aber auch hier sind technologische Verbesserungen zu erwarten. Praktisch wäre beispielsweise ein Gerät, das Bewegungsmuster aufzeichnet und damit Rückschlüsse auf psychische Zustände ermöglicht.»

\section{Der Arzt und der Roboter}

Ähnlich reagierte in Rüschlikon Andreas Meyer-Heim, Kinderarzt und medizinischer Leiter der Abteilung Rehabilitation am Universitäts-Kinderspital Zürich. Im Gespräch mit der SÄZ sagte er: «Besonders problematisch sind die versteckten psycho-mentalen Behinderungen, nach schweren Schädel-Hirn-Traumata beispielsweise. Da sind wir nicht selten hilflos. Und in diesem Bereich finden auch kaum Entwicklungen statt. Motorische Störungen werden eher wahrgenommen - und sind auch einfacher zu behandeln. Roboter werden hier zunehmend gute Dienste leisten: Sie ermöglichen eine bessere körperliche Funktion, und damit auch eine bessere gesellschaftliche Partizipation.» Illustratives Beispiel: «Ein Kind, das dank der Unterstützung eines Roboters ein paar Schritte gehen kann, kann leichter eine Schule besuchen, und ist dann auch besser integriert.» Roboter dürften in der Medizin also zunehmend auch bei behinderten Menschen erfolgreich einge- setzt werden. "Ganz wichtig dabei ist jedoch eine gute fächerübergreifende Kooperation. Ingenieure, Physio- und Ergotherapeutinnen müssen eng zusammenarbeiten, Neurologen, Pädiater und Biologen müssen sich eng vernetzen, um erfolgreich sein zu können.»

Fortschritte wünsche er sich insbesondere in der Neuropharmakologie, erklärte Meyer: «Dort sind wir praktisch noch nirgends. Schlucken oder sprechen können wir nur durch Üben verbessern. Schön wäre es, wenn wir ein Medikament einsetzen könnten, das die Neuroplastizität fördern würde.»

\section{Ökonomische und ethische Barrieren}

Die Studie des GDI zitiert die Präambel der Schweizerischen Bundesverfassung, wonach «die Stärke des Volkes sich misst am Wohl der Schwachen». «Im Vergleich zu früher geht es Menschen mit Behinderung in Europa heute sehr gut», heisst es in der Studie auch. Zunehmend aber würden Entwicklungen an ökonomische und an ethische Grenzen stossen. «Sparmassnahmen machen auch vor dem Sozialstaat nicht halt. Die verschiedenen Interessengruppen sind bemüht, ihr Lager vor solchen Kürzungen zu bewahren. Wenn jedoch nicht mehr alle Ansprüche berücksichtigt werden können, stellt sich die heikle Frage: Wer hat Recht auf wie viel?»

"Ja, es gibt unter den Behinderten Hierarchien", sagte gegenüber der SÄZ am Sitz des GDI der hörbehinderte Urs Germann. «Ein Lift ist hier selbst-

\section{«Psychische Behinderungen sind oft nicht sichtbar und deshalb besonders schwer $\mathrm{zu}$ verstehen.»}

verständlich. Eine Induktionsanlage jedoch gibt es nicht. Sie würde es Hörbehinderten erlauben, an dieser Veranstaltung teilzuhaben. Mit einem normalen Hörgerät hätte ich keine Chance, den Präsentationen folgen zu können. Nur weil ich persönlich das Glück habe, auf ein Cochlea-Implantat zählen zu dürfen, bekomme ich etwas mit.»

Wie gesagt: Es wurde schon viel gemacht und viel erreicht für behinderte Menschen. Gerade in diesem Land. Gleichzeitig gibt es noch einiges zu tun. Auch vor und hinter der eigenen Türe. 